\title{
Image-Guided Versus Blind Corticosteroid Injections in Adults With Shoulder Pain: A Systematic Review and Meta-Analysis
}

\author{
Peri Harish Kumar ${ }^{1}$, Tajbinder Singh Bains ${ }^{1}$, Naveen Shejale ${ }^{2}$, Varinder Kaur ${ }^{3}$ \\ 1. Department of Surgery, Armed Forces Medical College, Pune, IND 2. Department of Orthopedics, Indian Naval \\ Hospital Ship Asvini, Mumbai, IND 3. Department of Surgery, Adesh Institute of Medical Sciences and Research, \\ Bathinda, IND
}

Corresponding author: Peri Harish Kumar, kumarperiharish@gmail.com

\begin{abstract}
The present study aimed to compare the clinical effects of image-guided versus blind steroid injection to treat shoulder pain, which is always debatable. An electronic search of credible databases was conducted for randomized controlled trials (RCTs) comparing image-guided versus blind steroid injections. The continuous data were pooled as mean difference (MD) or standardized mean difference (SMD), and dichotomous data were grouped as odds ratio (OR) with $95 \%$ confidence interval (CI). Sensitivity analysis and leave-one-out analysis were performed. The meta-analysis of 20 RCTs comprising 1136 patients favored image-guided injection based on the MD of the visual analog score (VAS) and shoulder function scores, measured between six weeks follow-up and baseline $(\mathrm{MD}=0.63,95 \% \mathrm{CI}[0.13,1.12], \mathrm{p}=0.01$ and $\mathrm{SMD}=0.35,95 \% \mathrm{CI}[0.05,0.65]$, $\mathrm{p}=0.02$, respectively). Meta-analysis did not favor either group regarding the shoulder disability scores or side effects ( $M D=-2.18,95 \% \mathrm{CI}[-12.19,7.83], \mathrm{p}=0.67$, and $\mathrm{OR}=0.40,95 \% \mathrm{CI}[0.14,1.15], \mathrm{p}=0.09$, respectively). The image-guided approach was associated with significant improvement in pain and shoulder functionality. However, no significant difference was observed between the two approaches in terms of disability scores and side effects.
\end{abstract}

Review began $07 / 28 / 2021$ Review ended 08/01/2021 Published 08/09/2021

() Copyright 2021

Kumar et al. This is an open access article distributed under the terms of the Creative Commons Attribution License CC-BY 4.0., which permits unrestricted use, distribution, and reproduction in any medium, provided the original author and source are credited.
Categories: Pain Management, Physical Medicine \& Rehabilitation, Orthopedics

Keywords: corticosteroid, shoulder pain, blind injections, image-guided injection, meta-analysis

\section{Introduction And Background}

Shoulder pain can cause significant morbidity and compromise the person's ability to carry out daily activities and work [1-2]. The prevalence of shoulder disorders ranges from $7 \%$ to $36 \%$, and shoulder pain is prevalent in $16 \%$ to $26 \%$ of the population, and hence considered the third most common cause of musculoskeletal problems in primary care. Up to $1 \%$ of adults consult a general practitioner with the new incidence of shoulder pain annually [1,3]. Causes of shoulder pain include subacromial impingement syndrome, rotator cuff syndrome, subacromial bursitis, adhesive capsulitis, and biceps tendinitis [4].

Irrespective of the underlying etiology, glucocorticoid injections are widely used to treat shoulder pain and improve the functionality of shoulder movements. Two methods are used for drug delivery, a landmarkguided approach (i.e. blind) and an image-guided approach using ultrasonography [5]. The landmark-guided approach is ruled by factors such as local anatomy, distribution of peripheral fat, body mass index, different lengths of needles used, a suitable technique, and experience of the operator. Using ultrasound-guided steroid injection comes with the drawbacks of added cost, the need for an experienced operator, and it is time-consuming [6-7].

A comprehensive study is required to assess the evidence available and determine the best approach for managing shoulder pain. In our systematic review and meta-analysis, we investigated the efficacy and safety of image-guided versus blind corticosteroid injections in adults with shoulder pain.

\section{Review}

\section{Material and methods}

According to the Cochrane Handbook of Systematic Reviews of Interventions and the Preferred Reporting Items for Systematic Reviews and Meta-Analyses (PRISMA) statement guidelines, we carried out this study. No institutional review board approval was required for this systematic review.

Search Strategy

Relevant studies were identified through an extensive electronic search of databases such as PubMed, Web of Science, and Cochrane CENTRAL for reports till June 2021 from the inception. The search terms were (blind OR landmark OR anatomical OR image-guided OR ultrasound OR fluoroscopy) AND (steroid OR 
corticosteroid OR glucocorticoid OR triamcinolone OR methylprednisolone OR hydrocortisone OR prednisolone OR cortisone OR dexamethasone OR betamethasone) AND (frozen shoulder OR adhesive capsulitis OR shoulder pain OR shoulder impingement syndrome OR rotator cuff OR bursitis). Additionally, the reference lists of included studies were screened manually to identify additional articles, that are potentially relevant.

Study Selection

We included randomized controlled trials (RCTs) according to the PICO approach: P, patients with shoulder pain; I, ultrasound-guided corticosteroid (US-guided CS); C, comparison with blind or landmark injection of CS; and O, efficacy outcomes including changes of the visual analog score (VAS), shoulder function scores, shoulder abduction between baseline and six weeks follow-up, and safety outcomes included side effects. Exclusion criteria were observational studies, review articles, case reports, comments or guidelines, animal studies, non-English articles, and insufficient data to calculate. Disagreements were solved by consensus.

Data Extraction

For each paper, we extracted the following information: name of the first author, year of publication, country, number of patients, their mean age, percentage of females, type of shoulder disease, follow-up durations, and efficacy and safety outcomes.

Quality Assessment and Data Extraction

We used Cochrane Collaboration's tool for assessing the risk of bias of included RCTs [8]. Risk of bias assessment included the following domains: 1) sequence generation, 2) allocation sequence concealment, 3) blinding of participants and personnel, 4) blinding of outcome assessment, 5) incomplete outcome data, 6) selective outcome reporting, and 7) other potential sources of bias; the authors' judgment is categorized as 'Low risk,' 'High risk,' or 'Unclear risk' of bias.

Statistical Analysis

Continuous data were calculated as mean difference (MD) or standardized mean difference (SMD) with a 95\% confidence interval (CI). Dichotomous data were pooled as odds ratio (OR), with $95 \% \mathrm{CI}$. Heterogeneity was assessed by visual inspection of the forest plots and measured by Q statistics and statistics. Significant statistical heterogeneity was indicated by a Q statistic P-value less than 0.1 or by more than $50 \%$. In case of significant heterogeneity, a random effect model was employed. Otherwise, the fixed-effect model was used. All statistical tests were two-sided, and $\mathrm{P}<0.05$ was considered statistically significant. Leave-one-outmeta-analysis was performed to assess the contribution of each study to the overall model. The metaanalysis of all studies enrolled was conducted in OpenMeta[analyst] software.

\section{Results}

Search Strategy

A total of 2120 relevant papers were initially identified using the search strategy. A total of 324 duplicated papers were excluded by EndNote software, along with another 1750 papers that did not fit the criteria for analysis on the title and abstract screening. Based on full-text screening, 20 articles were finally included. The PRISMA flow process of study selection is shown in Figure 1. 


\section{Cureus}

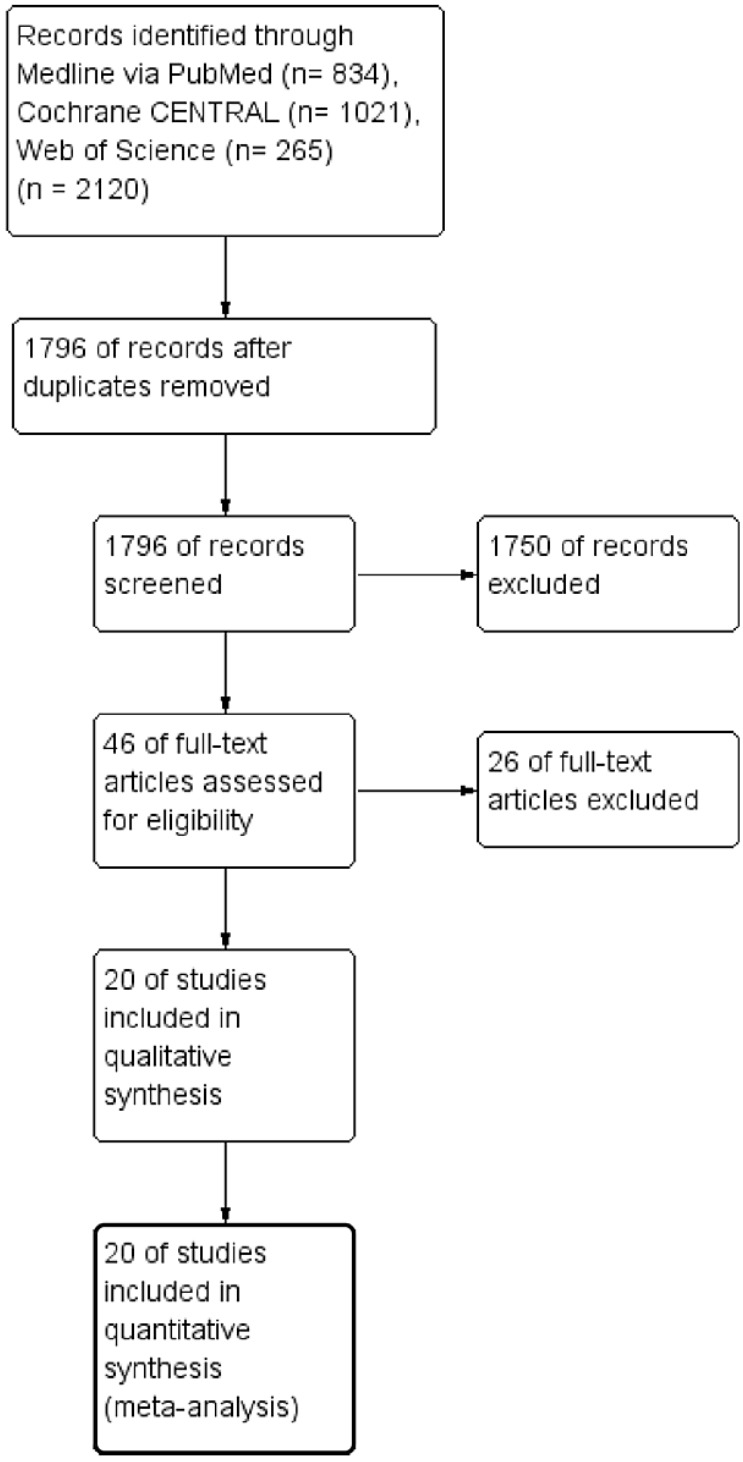

FIGURE 1: The PRISMA flow diagram of study selection

PRISMA: Preferred Reporting Items for Systematic Reviews and Meta-Analyses

Characteristics and Quality Assessment of the Included Studies

Ultimately, 20 RCTs, conducted in a total of 1136 patients, were included in this meta-analysis [9-28]. Of them, 581 patients had US-guided CS, and 555 had landmark CS injection. The patients included were diagnosed with subacromial impingement syndrome, rotator cuff syndrome, subacromial bursitis, adhesive capsulitis, and biceps tendonitis. All studies were published in English during the years from 2004 to 2021. Table 1 describes the significant characteristics of enrolled patients in each RCT. 


\begin{tabular}{|c|c|c|c|c|c|c|c|}
\hline Author & Year & $\begin{array}{l}\text { Country, and } \\
\text { study design }\end{array}$ & $\begin{array}{l}\text { Number of } \\
\text { patients }\end{array}$ & Age, mean & $\begin{array}{l}\% \text { of } \\
\text { Females }\end{array}$ & Diagnosis & $\begin{array}{l}\text { Follow-up } \\
\text { (weeks) }\end{array}$ \\
\hline Cho et al. [24] & 2021 & South Korea, RCT & T: 45; C: 45 & - & - & Primary frozen shoulder & 12 \\
\hline Akbari et al. [21] & 2020 & Turkey, RCT & T: 14; C: 14 & $\begin{array}{l}\mathrm{T}: 40.75 ; \mathrm{C}: \\
42.25\end{array}$ & $\begin{array}{l}\text { T: } 57.1 ; \mathrm{C}: \\
64.3\end{array}$ & $\begin{array}{l}\text { Subacromial impingement } \\
\text { syndrome }\end{array}$ & 4 \\
\hline $\begin{array}{l}\text { Yiannakopoulos } \\
\text { et al. [19] }\end{array}$ & 2020 & Greece, RCT & T: 22; C:22 & $\begin{array}{l}\mathrm{T}: 41.5 ; \mathrm{C}: \\
43.9\end{array}$ & $\begin{array}{l}\text { T: } 54.5 ; \mathrm{C}: \\
40.9\end{array}$ & Bicipital tendinosis & 6 \\
\hline $\begin{array}{l}\text { Raeissadat et al. } \\
\text { [28] }\end{array}$ & 2020 & Iran, $\mathrm{RCT}$ & T: 20; C: 21 & $\begin{array}{l}\text { T: } 57.8 ; \mathrm{C}: \\
59.9\end{array}$ & $\begin{array}{l}\mathrm{T}: 35 ; \mathrm{C}: \\
38.1\end{array}$ & $\begin{array}{l}\text { Shoulder adhesive } \\
\text { capsulitis }\end{array}$ & 4 \\
\hline Bhayana et al. [20] & 2018 & India, RCT & $\mathrm{T}: 30 ; \mathrm{C}: 30$ & $\begin{array}{l}\text { T: 44.53; C: } \\
42.03\end{array}$ & $\begin{array}{l}\text { T: } 56.6 ; \text { C: } \\
33.3\end{array}$ & Rotator cuff syndrome & 12 \\
\hline Coene et al. [9] & 2017 & USA, RCT & T: 9; C: 11 & 54 & $\begin{array}{l}\text { T: } 50 ; \mathrm{C}: \\
50\end{array}$ & $\begin{array}{l}\text { Shoulder adhesive } \\
\text { capsulitis }\end{array}$ & 12 \\
\hline Cole et al. [17] & 2015 & Australia, RCT & T: $28 ; \mathrm{C}: 28$ & T: 46; C:42 & $\begin{array}{l}\text { T: } 50 ; \mathrm{C}: \\
64\end{array}$ & $\begin{array}{l}\text { Subacromial impingement } \\
\text { syndrome }\end{array}$ & 6 \\
\hline $\begin{array}{l}\text { Haghighat et al. } \\
\text { [11] }\end{array}$ & 2015 & Iran, RCT & T: 20; C:20 & $\begin{array}{l}\text { T: } 50.45 ; \text { C: } \\
52.3\end{array}$ & $\begin{array}{l}\text { T: } 60 ; \mathrm{C}: \\
65\end{array}$ & $\begin{array}{l}\text { Subacromial impingement } \\
\text { syndrome }\end{array}$ & 6 \\
\hline Saeed et al. [18] & 2014 & Ireland, RCT & T: 50; C: 50 & 57.7 & 65 & $\begin{array}{l}\text { Subacromial impingement } \\
\text { syndrome }\end{array}$ & 12 \\
\hline Hsieh et al. [26] & 2013 & Taiwan, RCT & T: 46; C: 46 & $\begin{array}{l}\text { T: } 57.59 ; \mathrm{C}: \\
55.87\end{array}$ & $\begin{array}{l}\text { T: } 58.7 ; \mathrm{C}: \\
63\end{array}$ & Subacromial bursitis & 4 \\
\hline Dogu et al. [22] & 2012 & Turkey, RCT & T: 23; C: 23 & $\begin{array}{l}\text { T: } 55.17 ; \mathrm{C}: \\
56.74\end{array}$ & $\begin{array}{l}\mathrm{T}: 65.2 ; \mathrm{C}: \\
69.6\end{array}$ & $\begin{array}{l}\text { Subacromial impingement } \\
\text { syndrome }\end{array}$ & 6 \\
\hline Zufferey et al. [16] & 2012 & Switzerland, RCT & T: 32; C: 33 & T:53;C: 54 & $\begin{array}{l}1: 40.6 ; \mathrm{C}: \\
45.5\end{array}$ & Subacromial bursitis & 6 \\
\hline $\begin{array}{l}\text { Hashiuchi et al. } \\
\text { [15] }\end{array}$ & 2011 & Japan, RCT & T: 15; C: 15 & $\begin{array}{l}\text { T: } 59.7 ; \mathrm{C}: \\
67.8\end{array}$ & $\begin{array}{l}\text { T: } 46.7 ; \mathrm{C}: \\
66.7\end{array}$ & $\begin{array}{l}\text { Biceps tendon } \\
\text { inflammation }\end{array}$ & - \\
\hline Zhang et al. [14] & 2011 & China, RCT & T: 53; C: 45 & T: 49; C: 43 & $\begin{array}{l}\text { 1: } 35.8 ; \mathrm{C:} \\
35.6\end{array}$ & $\begin{array}{l}\text { Biceps tendon } \\
\text { inflammation }\end{array}$ & 32.5 \\
\hline $\begin{array}{l}\text { Panditaratne et al. } \\
\text { [12] }\end{array}$ & 2010 & $\begin{array}{l}\text { United Kingdom, } \\
\text { RCT }\end{array}$ & T: 41; C: 17 & 54 & 62.1 & Subacromial bursitis & 8 \\
\hline Ekeberg et al. [10] & 2009 & Norway, RCT & T: 53; C: 53 & T: $51 ; \mathrm{C}: 50$ & $\begin{array}{l}1: 60 ; \mathrm{C:} \\
62\end{array}$ & Rotator cuff disease & 6 \\
\hline Lee et al. [25] & 2009 & South Korea, RCT & T: 20; C: 20 & $\begin{array}{l}\text { T: } 53.1 ; \mathrm{C}: \\
54.1\end{array}$ & $\begin{array}{l}\text { T: } 55 ; \mathrm{C}: \\
50\end{array}$ & $\begin{array}{l}\text { Shoulder adhesive } \\
\text { capsulitis }\end{array}$ & 6 \\
\hline Ucuncu et al. [23] & 2009 & Turkey, RCT & T: 30; C: 30 & $\begin{array}{l}1: 52.1 ; \mathrm{C}: \\
52.9\end{array}$ & $\begin{array}{l}1: / 3.3 ; \mathrm{C}: \\
73.3\end{array}$ & Impingement syndrome & 6 \\
\hline Chen et al. [27] & 2006 & Taiwan, RCT & T: 20; C: 20 & 53 & 33.3 & Subacromial bursitis & 1 \\
\hline Gomoll et al. [6] & 2004 & Spain, RCT & T: 21; C: 2 & $\begin{array}{l}\text { T: } 52.9 ; \mathrm{C}: \\
51.9\end{array}$ & $\begin{array}{l}\text { T: } 71 ; \mathrm{C}: \\
60\end{array}$ & Impingement syndrome & 6 \\
\hline
\end{tabular}

TABLE 1: Summary table of studies included showing the significant characteristics of enrolled patients in each RCT

T: treatment; C: control; US-guided, ultrasound-guided; CS, corticosteroid; RCT, randomized controlled trial; DASH, the Disabilities of the Arm, Shoulder, and Hand; ASES, the American Shoulder and Elbow Surgeons score; CMS, Constant-Murley Score; ADLs, activities of daily living; SDQ, Shoulder Disability Questionnaire; SPADI, Shoulder Pain and Disability Index; SF-36, the 36-item Short-Form Health Survey 


\section{Cureus}

\begin{tabular}{|c|c|c|c|c|c|c|c|}
\hline & 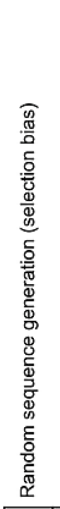 & 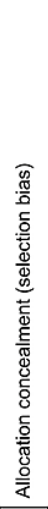 & 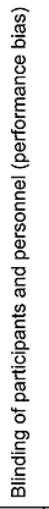 & 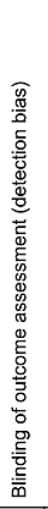 & 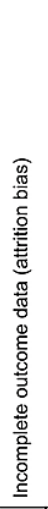 & 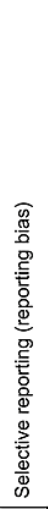 & 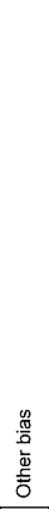 \\
\hline Akbari et al 2020 & $\odot$ & + & $?$ & $?$ & $\odot$ & $\odot$ & $\odot$ \\
\hline Bhayana et al 2018 & + & + & $?$ & $?$ & $\odot$ & + & + \\
\hline Chen et al 2006 & $\theta$ & $\theta$ & - & - & $\odot$ & $\theta$ & + \\
\hline Cho et al 2021 & + & + & + & + & $\odot$ & + & + \\
\hline Coene et al 2017 & $\odot$ & + & $?$ & $?$ & $\odot$ & + & $?$ \\
\hline Cole et al 2015 & + & + & + & $?$ & $\odot$ & + & + \\
\hline Dogu et al 2012 & + & + & + & $?$ & $\odot$ & + & + \\
\hline Ekeberg et al 2009 & + & + & $?$ & $?$ & $\odot$ & + & $?$ \\
\hline Haghighat et al 2015 & + & + & $?$ & $?$ & + & + & + \\
\hline Hashiuchi et al 2011 & + & + & $?$ & $?$ & $\odot$ & + & + \\
\hline Hsieh et al 2013 & + & + & $\ominus$ & $\odot$ & $\odot$ & + & + \\
\hline Lee et al 2009 & + & + & $?$ & + & + & + & + \\
\hline Naredo et al 2004 & $\odot$ & + & $?$ & $\odot$ & $\odot$ & + & + \\
\hline Panditaratne et al 2010 & $\theta$ & $\theta$ & $\theta$ & - & + & + & + \\
\hline Raeissadat et al 2017 & + & + & + & $?$ & + & + & + \\
\hline Saeed et al 2014 & + & + & $?$ & + & $\odot$ & + & + \\
\hline Ucuncu et al 2009 & + & + & - & + & $\odot$ & + & + \\
\hline Yiannakopoulos et al 2020 & $\odot$ & + & $?$ & $?$ & $\odot$ & + & + \\
\hline Zhang et al 2011 & $\odot$ & + & $?$ & $?$ & $\odot$ & + & + \\
\hline Zufferey et al 2012 & + & + & $\ominus$ & $\odot$ & $\odot$ & + & + \\
\hline
\end{tabular}

FIGURE 2: The summary of the risk of bias assessment of include studies

Included studies are Akbari et al. [21], Bhayana et al. [20], Chen et al. [27], Cho et al. [24], Coene et al. [9], Cole et al. [17], Dogu et al. [22], Ekeberg et al. [10], Gomoll et al. [6], Haghighat et al. [11], Hashiuchi et al. [15], Hsieh et al. [26], Lee et al. [25], Panditaratne et al. [12], Raeissadat et al. [28], Saeed et al. [18], Ucuncu et al. [23], Yiannakopoulos et al. [19], Zhang et al. [14], Zufferey et al. [16].

\section{Outcomes}

Visual Analogue Score (VAS)

Twelve studies reported data on changes in VAS scores between baseline and six weeks. Meta-analysis showed that US-guided CS injection provided significant reductions in VAS score than landmark injection $(\mathrm{MD}=0.63,95 \% \mathrm{CI}[0.13,1.12], \mathrm{p}=0.01)$. Significant heterogeneity was observed $\left(\mathrm{I}^{2}=85 \%, \mathrm{P}<0.001\right)$ (Figure 3 ). 


\section{Cureus}

\section{Studies}

Ucuncu et al 2009 Zufferey et al 2012 Dogu et al 2012

Hsieh et al 2013

Saeed et al 2014

Haghighat et al 2016

Panditaratne et al 201

Akbari et al 2020

Raeissadat et al 2017

Zhang et al 2011

Coene et al 2013

Overall (I^ $2=84.5 \%, P<0.001)$

Estimate (95\% C.I.)

$1.800 \quad(1.112, \quad 2.488)$

$1.300 \quad(0.566,2.034)$

$0.960 \quad(0.051,1.869)$

$-0.770(-2.161, \quad 0.621)$

$0.940 \quad(0.522,1.358)$

$0.400 \quad(0.175,0.625)$

$0.320(-0.801,1.441)$

$0.320(-0.801, \quad 1.441)$

$0.650(-0.616,1.916)$

$1.900(0.478,3.322)$

$-0.600(-0.936,-0.264)$

$0.000(-2.267,2.267)$

$0.627 \quad(0.134,1.120)$

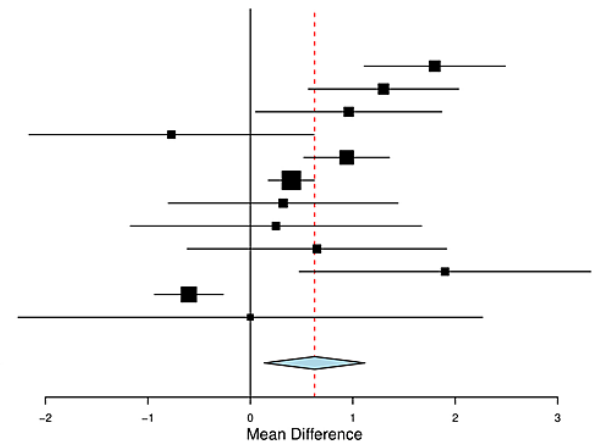

FIGURE 3: Forest plot of mean changes of visual analog score (VAS) between baseline and six weeks of image-guided vs blind steroid injection

Included studies are: Dogu et al. [22], Ucuncu et al. [23], Zufferey et al. [16]. Haghighat et al. [11], Hsieh et al. [26], Saeed et al. [18], Akbari et al. [21], Panditaratne et al. [12], Raeissadat et al. [28], Coene et al. [9], Lee et al. [25], Zhang et al. [14]

Shoulder Function Scores

Nine studies reported on changes in shoulder function scores between baseline and six weeks. Four of them used the Constant-Murley Score, two studies used the Oxford score, and three studies used other scores. Meta-analysis showed that US-guided CS was associated with better shoulder function scores at six weeks than landmark CS $(\mathrm{SMD}=0.35,95 \% \mathrm{CI}[0.05,0.65], \mathrm{p}=0.02)$ as shown in Figure $4 a$. Significant heterogeneity was observed $\left(\mathrm{I}^{2}=61 \%, \mathrm{p}=0.008\right)$, which was resolved after excluding the study by Ucuncu et al. (2009) $\left(\mathrm{SMD}=0.24,95 \% \mathrm{CI}[0.03,0.45], \mathrm{p}=0.03 ; \mathrm{I}^{2}=15 \%, \mathrm{p}=0.31\right)$, as shown in Figure $4 b$. 


\section{Cureus}

\section{Studies}

Ucuncu et al 2009 Akbari et al 2020

hang 2020

ren 2018

Bhayana et al 2018

Panditaratne et al 2010

Ceet al 2009

Cole et al 2015

Overall (1^2 $2=61.27 \%, P=0.008)$
Estimate (95: C.I.)

$1.307 \quad(0.749,1.864)$

$\begin{array}{lr}1.307 & (0.749,1.864) \\ 0.314 & (-0.431,1.060)\end{array}$

$0.537(0.133,0.942)$

$0.121(-0.386,0.627)$

$-0.121(-0.686,0.445)$

$0.095(-0.517,0.708)$

$0.095(-0.517,0.708)$

$0.094(-0.526,0.71$

$0.815(-0.178,1.452)$

$0.353(0.052,0.654)$
Figure 4a

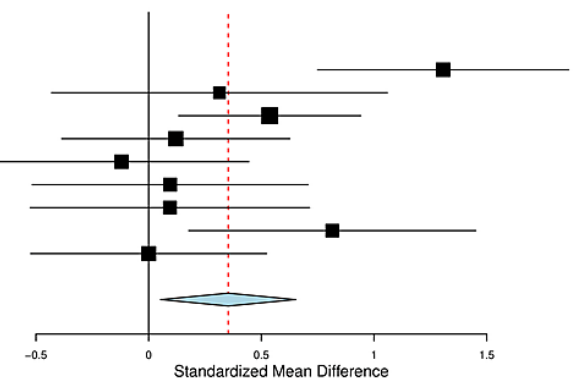

Figure $4 b$

Studies

Estimate (95\% C.I.)

Akbari et al 2020

Zhang et al 2011

Bhayana et al 2018

Panditaratne et al 2010

Raeissadat et al 2017

Lee et al 2009

Naredo et al 2004

$0.314(-0.431,1.060)$

$\begin{array}{lrl}0.314 & (-0.431, & 1.060) \\ 0.537 & (0.133, & 0.942)\end{array}$

$\begin{array}{lrl}0.537 & (0.133, & 0.942) \\ 0.121 & (-0.386, & 0.627)\end{array}$

$\begin{array}{ccc}0.537 & (0.133, & 0.942) \\ -0.121 & (-0.386, & 0.627) \\ 0.086, & 0.445)\end{array}$

$0.095(-0.517,0.708)$

$0.094(-0.526,0.715)$ $0.815 \quad(0.178,1.452)$

$0.000(-0.524,0.524)$

$0.239(0.026,0.453)$

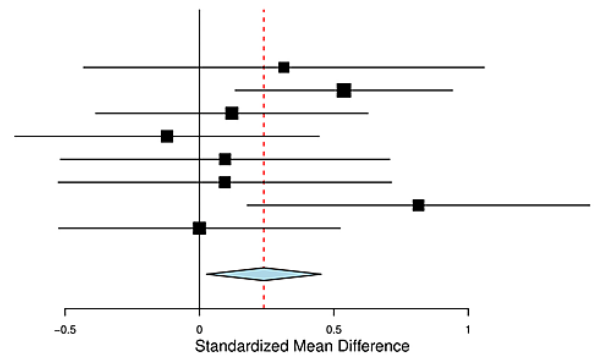

FIGURE 4: Forest plots of (a) mean changes and (b) sensitivity analysis of mean changes of shoulder function scores between baseline and six weeks of image-guided vs blind steroid injection.

Included studies are: Akbari et al. [21], Bhayana et al. [20], Cole et al. [17], Gomoll et al. [6], Lee et al. [25], Panditaratne et al. [12], Raeissadat et al. [28], Ucuncu et al. [23], Zhang et al. [14].

Shoulder Pain and Disability Index (SPADI)

Three studies reported on changes of SPADI at the six weeks follow-up. Meta-analysis did not favor either group ( $\mathrm{MD}=-2.18$, 95\% CI [-12.19, 7.83], p=0.67), as represented in Figure 5a. Significant heterogeneity was observed $\left(\mathrm{I}^{2}=77 \%, \mathrm{P}<0.012\right)$. Heterogeneity was resolved after excluding the study by Hsieh et al. (2013) $\left(\mathrm{MD}=-7.9,95 \% \mathrm{CI}[-9.29,-6.15], \mathrm{P}<0.001 ; \mathrm{I}^{2}=0 \%, \mathrm{p}=0.59\right)$, which is shown in Figure $5 b$. 


\section{Cureus}

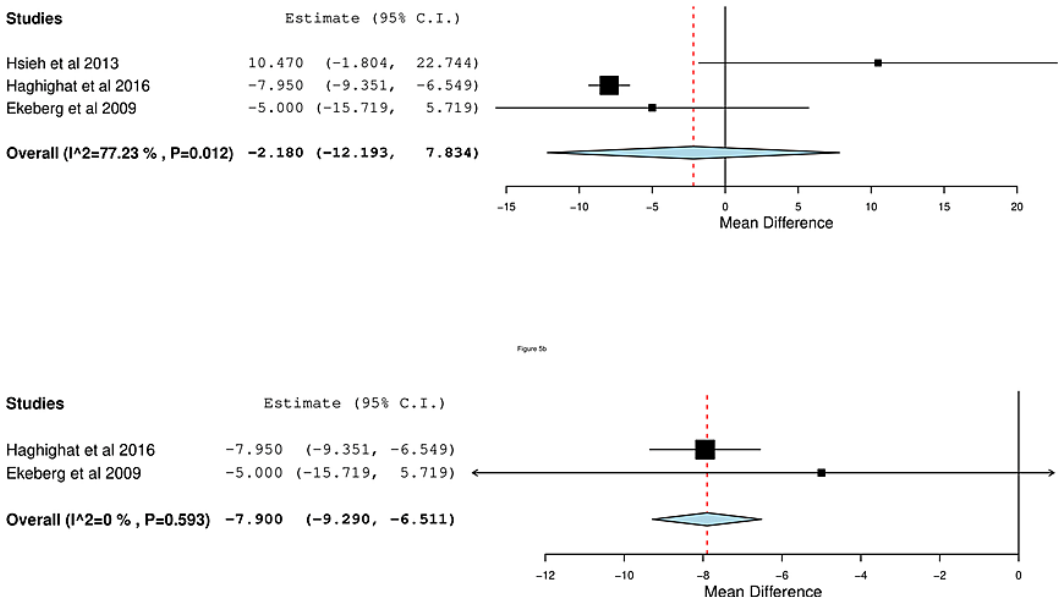

FIGURE 5: Forest plots of (a) mean changes of SPADI and (b) sensitivity analysis of mean changes of SPADI between baseline and six weeks of image-guided vs blind steroid injection.

Included studies are: Ekeberg et al. [10], Haghighat et al. [11], Hsieh et al. [26].

Shoulder Abduction Degree

Eleven studies reported on changes in shoulder abduction at six weeks. Meta-analysis showed that USguided CS was associated with larger shoulder abduction than landmark CS (MD=6.07, 95\% CI [3.95, 14.19], P $<0.001)$. Significant heterogeneity was observed $\left(\mathrm{I}^{2}=95 \%, \mathrm{P}<0.001\right)$ and is shown in Figure 6.

\begin{tabular}{lrrr} 
Studies & \multicolumn{3}{c}{ Estimate $(95 \%$ C.I.) } \\
& & & \\
Ucuncu et al 2009 & 20.300 & $(9.327$, & $31.273)$ \\
Dogue et al 2012 & 1.570 & $(0.701$, & $2.439)$ \\
Hsieh et al 2013 & 5.710 & $(1.666$, & $9.754)$ \\
Haghighat et al 2016 & 6.500 & $(4.502$, & $8.498)$ \\
Akbari et al 2020 & -7.500 & $(-24.012$, & $9.012)$ \\
Raeissadat et al 2017 & 1.730 & $(-7.583$, & $11.043)$ \\
Lee et al 2009 & -2.800 & $(-7.209$, & $1.609)$ \\
Naredo et al 2004 & 9.400 & $(2.564,16.236)$ \\
Chen et al 2006 & 41.270 & $(34.779$, & $47.761)$ \\
Ekeberg et al 2009 & 12.250 & $(5.176,19.324)$ \\
Coene et al 2013 & 8.300 & $(-3.131,19.731)$ \\
Overall (I^2=94.62\%, P< 0.001) & $\mathbf{9 . 0 6 9}$ & $(3.948,14.190)$
\end{tabular}

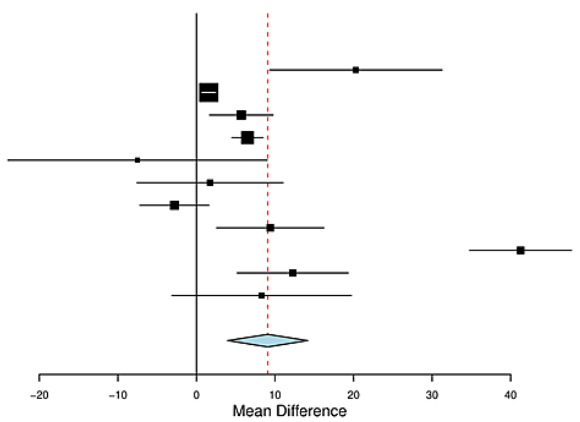

FIGURE 6: Forest plot of mean changes of shoulder abduction degree between baseline and six weeks of image-guided vs blind steroid injection.

Included studies are: Akbari et al. [21], Coene et al. [9], Dogu et al. [22], Ekeberg et al. [10], Haghighat et al. [11], Hsieh et al. [26], Lee et al. [25], Raeissadat et al. [28], Ucuncu et al. [23], Gomoll et al. [6].

Adverse Events

Nine studies have reported on adverse events, including infection and skin peeling. Meta-analysis showed no significant difference between the compared groups (OR=0.40, 95\% CI [0.14, 1.15], $\mathrm{p}=0.09)$, and significant heterogeneity was observed $\left(\mathrm{I}^{2}=0 \%, \mathrm{p}=0.97\right)$, and are shown in Figure 7 . 


\section{Cureus}

Studies

Ucuncu et al 2009

Dogu et al 2012

Raeissadat et al 2017

Naredo et al 2004

hannakopous et al 202

Cho et al 2021

Heshiuchi 212011

Hashiuchi et al 2011
Ekeberg et al 2009

Overall $(1 \wedge 2=0 \%, P=0.966) \quad 0.399 \quad(0.138, \quad 1.152)$ $1.000(0.019,52.531)$ $1.049(0.020,55.366) \quad 0 / 20$ $0.302(0.012,5.066) \quad 0 / 21$ $1.000(0.019,52.036)$ 0.30 $1.000(0.019,51.491) \quad 0 / 45$ $1.000(0.019,53.659) \quad 0 / 15$ $0.236 \quad(0.025,2.182) \quad 1 / 53$

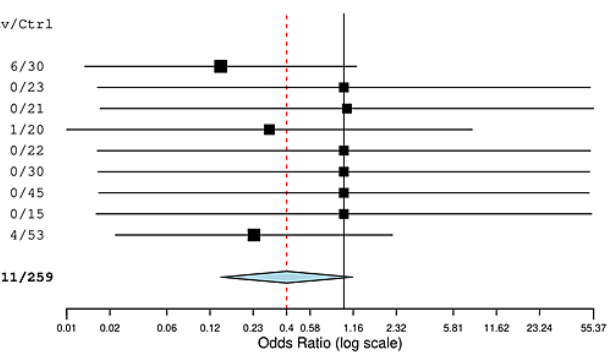

FIGURE 7: Forest plot of side effects of image-guided vs blind steroid injection

Included studies are: Dogu et al. [22], Raeissadat et al. [28], Ucuncu et al. [23], Bhayana et al. [20], Cho et al. [24], Ekeberg et al. [10], Gomoll et al. [6], Hashiuchi et al. [15], Yiannakopoulos et al. [19].

Leave-One-Out-Analysis

Significant heterogeneity was observed in most outcomes $\left(\mathrm{I}^{2}>50 \%, \mathrm{P}<0.1\right)$; therefore, a random-effects model was utilized. We conducted a leave-one-out meta-analysis to assess the contribution of each study to the overall model. No study affected the overall estimate in the leave-one-out-study analysis, and there was no marked difference in results, suggesting that the results of the current study were not driven by a single study or a supplementary file. The Forest plot of the leave-one-out meta-analysis of the visual analog score is shown in Figure 8.

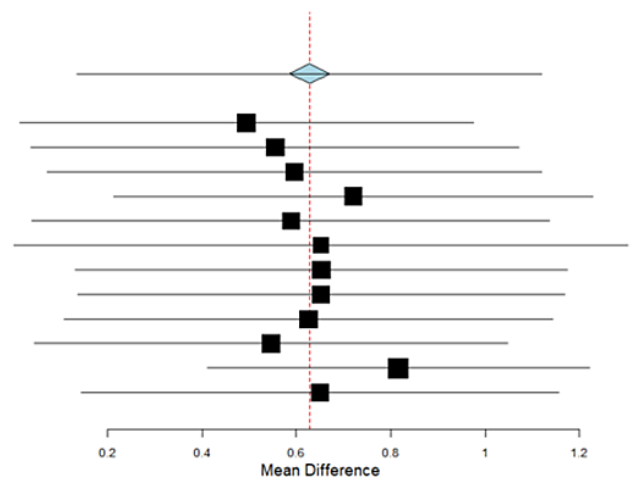

Forest plot of leave-one-out meta-analysis of visual analogue score

FIGURE 8: Forest plot of leave-one-out meta-analysis of the visual analog score

Included studies are: Dogu et al. [22], Ucuncu et al. [23], Zufferey et al. [16]. Haghighat et al. [11], Hsieh et al. [26], Saeed et al. [18], Akbari et al. [21], Panditaratne et al. [12], Raeissadat et al. [28], Coene et al. [9], Lee et al. [25], Zhang et al. [14].

Figure 9 represents the Forest plot of the leave-one-out meta-analysis of shoulder function scores. 


\section{Cureus}

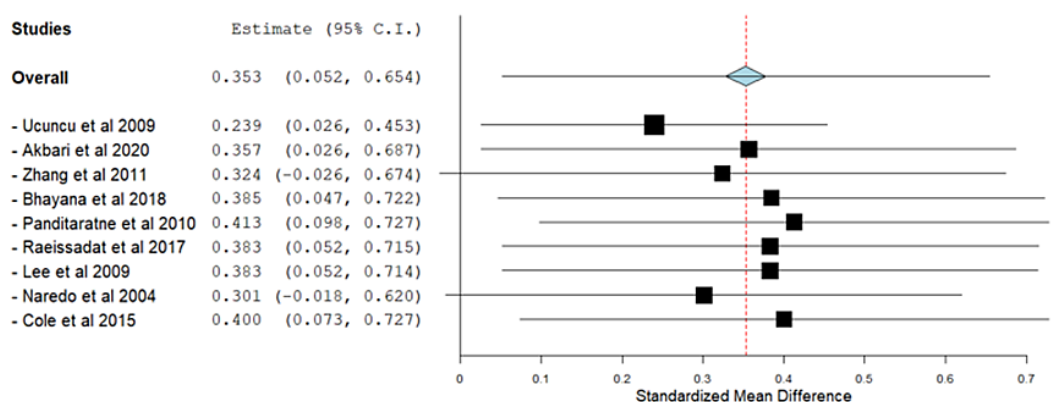

Forest plot of leave-one-out meta-analysis of shoulder function scores

FIGURE 9: Forest plot of leave-one-out meta-analysis of shoulder function scores.

Included studies are: Akbari et al. [21], Bhayana et al. [20], Cole et al. [17], Gomoll et al. [6], Lee et al. [25], Panditaratne et al. [12], Raeissadat et al. [28], Ucuncu et al. [23], Zhang et al. [14].

As discussed, the Forest plot of leave-one-out meta-analysis of shoulder abduction can be found in Figure 10 .

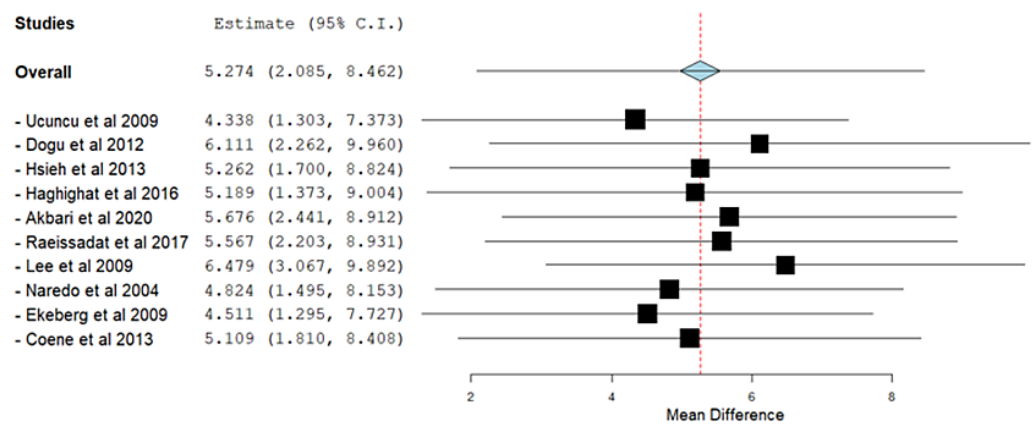

Forest plot of leave-one-out meta-analysis of shoulder abduction

FIGURE 10: Forest plot of the leave-one-out meta-analysis of shoulder abduction

Included studies are: Akbari et al. [21], Coene et al. [9], Dogu et al. [22], Ekeberg et al. [10], Haghighat et al. [11], Hsieh et al. [26], Lee et al. [25], Raeissadat et al. [28], Ucuncu et al. [23], Gomoll et al. [6].

Figure 11 shows the Forest plot of the leave-one-out meta-analysis of adverse events. 


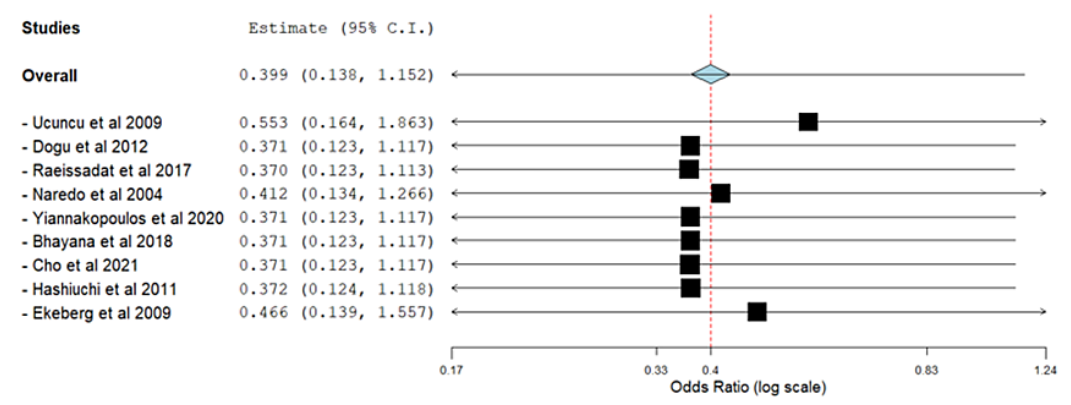

Forest plot of leave-one-out meta-analysis of adverse events

\section{FIGURE 11: Forest plot of the leave-one-out meta-analysis of adverse} events

Included studies are: Dogu et al. [22], Raeissadat et al. [28], Ucuncu et al. [23], Bhayana et al. [20], Cho et al. [24], Ekeberg et al. [10], Gomoll et al. [6], Hashiuchi et al. [15], Yiannakopoulos et al. [19].

\section{Discussion}

The current meta-analysis showed that image-guided steroid injection was associated with a definite improvement in pain and shoulder functionality. However, the difference between the two approaches was not significant regarding disability scores and side effects.

Our results are concordant with two previously reported meta-analyses [29-30]. A meta-analysis by Aly et al. reported their results based on three studies for the subacromial space and found that the image-guided group had a significant reduction in pain compared to the landmark-guided (LMG) group at six weeks postinjection [30]. Intriguingly, the accuracy of needle injection was similar between the two groups (70\% in the blind injection group and $65 \%$ in the image-guided group). On the other hand, improved accuracy of the glenohumeral joint and biceps tendon sheath injections was associated with an improved clinical outcome in the US group [30]. Another meta-analysis by Bloom et al. included three studies that reported pain improvement in the image-guided group compared to the blind injection group. There was significant heterogeneity, and when the sensitivity analysis is carried out, the benefit was no longer apparent [29].

Many scores assess functional outcomes of the shoulder, and our analysis showed that image-guided steroid injection was resulted in better shoulder function scores at six weeks compared to blind steroid injection. Aly et al. reported an improvement in the shoulder function in the image-guided group at six weeks postinjection compared to the blind injection group. However, there was significant heterogeneity among the studies included [30]. Our results also showed a substantial heterogeneity among the included trials, which was best resolved after excluding the study by Ucuncu et al. [13]. There are many reasons for heterogeneity such as different sources of shoulder pain and different durations of follow-up, inadequate patient blinding that may be vulnerable to bias, and possible placebo effects. Also, most of the studies included patients with chronic diseases, which might have impacted clinical outcomes substantially. In a study by Ucuncu et al., the design was not blinded, and placebo effect expectation was higher in the imageguided group. The study also reported that patients included were not restricted to use anti-inflammatory drugs, thus impacting clinical outcomes [13].

We evaluated changes in shoulder abduction at the sixth week, and our results indicated that image-guided steroid injection was associated with more extensive shoulder abduction than blind injection. Blooms et al. reported a difference between groups, favoring the image-guided group at one to two weeks after injection [29]. We evaluated adverse events in both groups regarding the safety profile and found no significant difference between the compared groups.

\section{Strengths and limitations}

Our study included large randomized controlled trials with moderate to high quality and large sample size, helping to understand better and evaluate the evidence clearly. We strictly adhered to the PRISMA checklist and carefully performed a precise search in the electronic database. Moreover, the mean changes between baseline and six weeks of follow-up were calculated, which offered a more accurate detection of changes at 
baseline. The limitations in our study included: 1) a substantial heterogeneity was observed in our analysis $\left(\mathrm{I}^{2}>50 \%, \mathrm{P}<0.1\right)$, and we conducted a leave-one-out meta-analysis to assess the contribution of each study to the overall model. No study affected the overall estimate in the leave-one-out-study analysis, and there was no marked difference in results, suggesting that a single study did not drive the results of the current study. 2) There was marked variation in the functional scales used between studies (Constant-Murley Score, Oxford score, and other scores); therefore, we used SMD for all functional scores. 3) Due to the inadequate data in the literature, the correlation between injection accuracy and clinical improvement was not studied.

\section{Conclusions}

Our meta-analysis investigated the efficacy and safety of image-guided injections compared to blind steroid injections. In terms of efficacy, the image-guided approach was associated with significant improvement in pain and shoulder functionality. However, we found no significant difference between the two approaches in terms of safety. Future studies recommend investigating and comparing the risk of adverse events associated with these approaches and correlate needle injection accuracy and the efficacy and safety of the outcomes. Also, further studies to assess the impact of operators (radiologists or non-radiologists) on the outcomes and cost-effectiveness of the two approaches are desired.

\section{Additional Information \\ Disclosures}

Conflicts of interest: In compliance with the ICMJE uniform disclosure form, all authors declare the following: Payment/services info: All authors have declared that no financial support was received from any organization for the submitted work. Financial relationships: All authors have declared that they have no financial relationships at present or within the previous three years with any organizations that might have an interest in the submitted work. Other relationships: All authors have declared that there are no other relationships or activities that could appear to have influenced the submitted work.

\section{Acknowledgements}

We state that the authors Peri Harish Kumar and Tajbinder Singh Bains have contributed equally to this paper and hence share First Authorship.

\section{References}

1. Saper MG, Pierpoint LA, Liu W, Comstock RD, Polousky JD, Andrews JR: Epidemiology of shoulder and elbow injuries among United States high school baseball players: school years 2005-2006 through 2014-2015. Am J Sports Med. 2018, 46:37-43. 10.1177/0363546517734172

2. Meislin RJ, Sperling JW, Stitik TP: Persistent shoulder pain: epidemiology, pathophysiology, and diagnosis . Am J Orthop (Belle Mead NJ). 2005, 34:5-9.

3. Mitchell C, Adebajo A, Hay E, Carr A: Shoulder pain: diagnosis and management in primary care . BMJ. 2005, 331:1124-8. 10.1136/bmj.331.7525.1124

4. Greenberg DL: Evaluation and treatment of shoulder pain . Med Clin North Am. 2014, 98:487-504. 10.1016/j.mcna.2014.01.016

5. Soh E, Li W, Ong KO, Chen W, Bautista D: Image-guided versus blind corticosteroid injections in adults with shoulder pain: a systematic review. BMC Musculoskelet Disord. 2011, 12:137. 10.1186/1471-2474-12-137

6. Gomoll AH, Katz JN, Warner JJ, Millett PJ: Rotator cuff disorders: recognition and management among patients with shoulder pain. Arthritis Rheum. 2004, 50:3751-61. 10.1002/art.20668

7. Xiao RC, Walley KC, DeAngelis JP, Ramappa AJ: Corticosteroid injections for adhesive capsulitis. A review . Clin J Sport Med. 2017, 27:308-20. 10.1097/JSM.0000000000000358

8. Higgins JPT, Thomas J, Chandler J, Cumpston M, Li T, Page MJ, Welch VA: Cochrane Handbook for Systematic Reviews of Interventions. John Wiley \& Sons, Hoboken, New Jersey; 2019. 10.1002/9781119536604

9. Comparison of pain relief and functional improvement in landmark vs. ultrasound-guided corticosteroid injections for adhesive capsulitis: a prospective study. (2017).

https://www.proquest.com/openview/7bcd73b057bbbb0561d231766ce60d53/1?pqorigsite $=$ gscholar $\&$ cbl $=18750 \&$ diss $=$.

10. Ekeberg OM, Bautz-Holter E, Tveitå EK, Juel NG, Kvalheim S, Brox JI: Subacromial ultrasound guided or systemic steroid injection for rotator cuff disease: randomised double blind study. BMJ. 2009, 338:a3112. 10.1136/bmj.a3112

11. Haghighat S, Taheri P, Banimehdi M, Taghavi A: Effectiveness of blind \& ultrasound guided corticosteroid injection in impingement syndrome. Glob J Health Sci. 2015, 8:179-84. 10.5539/gjhs.v8n7p179

12. Panditaratne N, Wilkinson C, Groves C, Chandramohan M: Subacromial impingement syndrome: a prospective comparison of ultrasound-guided versus unguided injection techniques. Ultrasound. 2010, 18:176-81. 10.1258/ult.2010.010022

13. Campisi CC, Larcher L, Lavagno R, et al.: Microsurgical primary prevention of lymphatic injuries following breast cancer treatment. Plast Reconstr Surg. 2012, 130:749e-50e. 10.1097/PRS.0b013e318267d906

14. Zhang J, Ebraheim N, Lause GE: Ultrasound-guided injection for the biceps brachii tendinitis: results and experience. Ultrasound Med Biol. 2011, 37:729-33. 10.1016/j.ultrasmedbio.2011.02.014

15. Hashiuchi T, Sakurai G, Morimoto M, Komei T, Takakura Y, Tanaka Y: Accuracy of the biceps tendon sheath injection: ultrasound-guided or unguided injection? A randomized controlled trial. J Shoulder Elbow Surg. 
2011, 20:1069-73. 10.1016/j.jse.2011.04.004

16. Zufferey P, Revaz S, Degailler X, Balague F, So A: A controlled trial of the benefits of ultrasound-guided steroid injection for shoulder pain. Joint Bone Spine. 2012, 79:166-9. 10.1016/j.jbspin.2011.04.001

17. Cole BF, Peters KS, Hackett L, Murrell GA: Ultrasound-guided versus blind subacromial corticosteroid injections for subacromial impingement syndrome: a randomized, double-blind clinical trial. Am J Sports Med. 2016, 44:702-7. 10.1177/0363546515618653

18. Saeed A, Khan M, Morrissey S, Kane D, Fraser AD: Impact of outpatient clinic ultrasound imaging in the diagnosis and treatment for shoulder impingement: a randomized prospective study. Rheumatol Int. 2014, 34:503-9. 10.1007/s00296-013-2892-z

19. Yiannakopoulos CK, Megaloikonomos PD, Foufa K, Gliatis J: Ultrasound-guided versus palpation-guided corticosteroid injections for tendinosis of the long head of the biceps: a randomized comparative study. Skeletal Radiol. 2020, 49:585-91. 10.1007/s00256-019-03315-9

20. Bhayana H, Mishra P, Tandon A, Pankaj A, Pandey R, Malhotra R: Ultrasound guided versus landmark guided corticosteroid injection in patients with rotator cuff syndrome: randomised controlled trial. J Clin Orthop Trauma. 2018, 9:S80-5. 10.1016/j.jcot.2017.01.005

21. Akbari N, Ozen S, Şenlikçi HB, Haberal M, Çetin N: Ultrasound-guided versus blind subacromial corticosteroid and local anesthetic injection in the treatment of subacromial impingement syndrome: a randomized study of efficacy. Jt Dis Relat Surg. 2020, 31:115-22. 10.5606/ehc.2020.71056

22. Dogu B, Yucel SD, Sag SY, Bankaoglu M, Kuran B: Blind or ultrasound-guided corticosteroid injections and short-term response in subacromial impingement syndrome. A randomized, double-blind, prospective study. Am J Phys Med Rehabil. 2012, 91:658-65. 10.1097/PHM.0b013e318255978a

23. Ucuncu F, Capkin E, Karkucak M, Ozden G, Cakirbay H, Tosun M, Guler M: A comparison of the effectiveness of landmark-guided injections and ultrasonography guided injections for shoulder pain. Clin J Pain. 2009, 25:786-9. 10.1097/AJP.0b013e3181acb0e4

24. Cho CH, Min BW, Bae KC, Lee KJ, Kim DH: A prospective double-blind randomized trial on ultrasoundguided versus blind intra-articular corticosteroid injections for primary frozen shoulder. Bone Joint J. 2021, 103-B:353-9. 10.1302/0301-620X.103B2.BJJ-2020-0755.R1

25. Lee HJ, Lim KB, Kim DY, Lee KT: Randomized controlled trial for efficacy of intra-articular injection for adhesive capsulitis: ultrasonography-guided versus blind technique. Arch Phys Med Rehabil. 2009, 90:19972002. 10.1016/j.apmr.2009.07.025

26. Hsieh LF, Hsu WC, Lin YJ, Wu SH, Chang KC, Chang HL: Is ultrasound-guided injection more effective in chronic subacromial bursitis?. Med Sci Sports Exerc. 2013, 45:2205-13. 10.1249/MSS.0b013e31829b183c

27. Chen MJ, Lew HL, Hsu TC, et al.: Ultrasound-guided shoulder injections in the treatment of subacromial bursitis. Am J Phys Med Rehabil. 2006, 85:31-5. 10.1097/01.phm.0000184158.85689.5e

28. Raeissadat SA, Nouri F, Darvish M, Esmaily H, Ghazihosseini P: Ultrasound-guided injection of high molecular weight hyaluronic acid versus corticosteroid in management of plantar fasciitis: a 24-week randomized clinical trial. J Pain Res. 2020, 13:109-21. 10.2147/JPR.S217419

29. Bloom JE, Rischin A, Johnston RV, Buchbinder R: Image-guided versus blind glucocorticoid injection for shoulder pain. Cochrane Database Syst Rev. 2012, 2012:CD009147.

30. Aly AR, Rajasekaran S, Ashworth N: Ultrasound-guided shoulder girdle injections are more accurate and more effective than landmark-guided injections: a systematic review and meta-analysis. Br J Sports Med. 2015, 49:1042-9. 10.1136/bjsports-2014-093573 